\title{
Adjuvant therapy in invasive intraductal papillary mucinous neoplasm (IPMN) of the pancreas: a systematic review
}

\author{
Linus Aronsson, Sofia Marinko, Daniel Ansari, Roland Andersson \\ Department of Clinical Sciences Lund, Surgery, Lund University, Skåne University Hospital, Lund, Sweden \\ Contributions: (I) Conception and design: R Andersson; (II) Administrative support: All authors; (III) Provision of study materials or patients: L \\ Aronsson, S Marinko; (IV) Collection and assembly of data: L Aronsson, S Marinko; (V) Data analysis and interpretation: L Aronsson, S Marinko, D \\ Ansari; (VI) Manuscript writing: All authors; (VII) Final approval of manuscript: All authors. \\ Correspondence to: Roland Andersson, MD, PhD. Department of Surgery, Clinical Science Lund, Lund University, Skåne University Hospital, SE-221 \\ 85 Lund, Sweden. Email: roland.andersson@med.lu.se.
}

\begin{abstract}
Intraductal papillary mucinous neoplasm (IPMN) of the pancreas is a cystic tumor with a disease spectrum ranging from low-grade dysplasia to invasive carcinoma. The evidence for adjuvant treatment in invasive IPMN is limited and mostly derived from studies in conventional pancreatic ductal adenocarcinoma (PDAC). We performed a systematic review focusing on all clinical studies concerning the efficacy of adjuvant therapy in patients with invasive IPMN. We identified 8 retrospective cohort studies, using either adjuvant chemotherapy alone $(n=1)$, adjuvant radiotherapy alone $(n=1)$ or adjuvant chemotherapy in combination with radiation $(n=6)$. Adjuvant therapy was associated with a survival benefit in 7 out of the 8 studies. Specific survival benefit was noted for patients with node-positive disease, higher TNM stage, positive resection margins, poor differentiation and tubular subtype. We conclude that adjuvant therapy may be beneficial in invasive IPMN, but current data suggest that it should be given selectively based on individual tumor characteristics. Further prospective, randomized studies are warranted.
\end{abstract}

Keywords: Invasive intraductal papillary mucinous neoplasm (invasive IPMN); adjuvant therapy; lymph node metastasis; tubular differentiation; survival

Submitted Jun 27, 2019. Accepted for publication Sep 11, 2019.

doi: $10.21037 /$ atm.2019.10.37

View this article at: http://dx.doi.org/10.21037/atm.2019.10.37

\section{Background}

Intraductal papillary mucinous neoplasm (IPMN) of the pancreas is a cystic tumor arising from the cells lining the pancreatic ducts. IPMN is divided into main-duct (MD), branch-duct (BD), and mixed-type (MT) lesions depending on the site of origin (1). The disease spectrum ranges from low-grade dysplasia to invasive carcinoma (2). The treatment is surgical, and the preoperative assessment is aided by guidelines outlining specific findings to distinguish IPMNs with a high risk of being malignant and thus surgical candidates from the benign ones that can undergo initial surveillance $(3,4)$. The prognosis following surgical resection of IPMN with an associated invasive carcinoma has been reported better as compared to 'ordinary' pancreatic ductal adenocarcinoma (PDAC) (5).

The invasive carcinoma component can be classified into histological subtypes where the tubular, colloid and oncocytic subtypes are the most common (2). There seems to be an underlying difference in tumor biology between the different subtypes (6-8). A more favorable prognosis is reported for the colloid and oncocytic subtypes, while the tubular subtype has a survival rate comparable to PDAC $(9,10)$. The most recent guidelines recommend adjuvant therapy for IPMN with an associated invasive carcinoma (3). However, the literature is sparse as regards the type of regimen and outcome $(3,4)$.

For PDAC, there is extensive evidence that supports the use of adjuvant chemotherapy following resection. The estimated 5 -year survival rate for PDAC patients receiving adjuvant 
chemotherapy is up to $30 \%(11-13)$, in contrast to about $8 \%$ in patients not receiving adjuvant chemotherapy (11).

As the data concerning adjuvant therapy for invasive IPMN have not been previously collated and synthesized, we performed a systematic review of all available literature to assess whether adjuvant therapy improves outcome in these patients.

\section{Methods}

A systematic review was conducted in accordance with the PRISMA statement (14). The literature search was independently conducted by Sofia Marinko and Linus Aronsson. No ethical approval was necessary for this study due to the exclusive use of secondary data.

\section{Search strategy and study selection}

Electronic searches of the PubMed, Embase, and Cochrane Library databases were performed to identify articles regarding the efficacy of adjuvant therapy following resection in IPMN with an associated invasive carcinoma (henceforth referred to as "invasive IPMN"). Searches included articles published within the last 10 years, up until January 1, 2019.

The criteria for inclusion were the following: (I) cohort study with resected, histopathologically confirmed invasive IPMN, (II) no neoadjuvant therapy, (III) administration of adjuvant chemotherapy and/or radiotherapy, (IV) reporting of overall survival (OS), cancer-specific survival (CSS) or disease-free survival (DFS). Case reports, conference abstracts and reviews were excluded. Only publications written in the English language were considered. The full texts of eligible articles were thoroughly reviewed and their quality and relevance for this study were assessed.

\section{Data extraction and presentation}

Data on number of participants, chemotherapy regimen, survival and main findings were tabulated and compared between the included studies. The primary endpoint was CSS when available, otherwise OS was used.

\section{Results}

The literature search yielded 143 publications. Following screening of title, abstracts and full text, eight articles (15-22) were considered eligible for inclusion (Figure 1). All studies were retrospective in nature. The cohort size ranged from 44 to 1,220 patients. All patients underwent resection for a histopathologically confirmed invasive IPMN. Adjuvant chemotherapy was evaluated in one study (16), adjuvant radiotherapy in one study (19), while six studies evaluated adjuvant chemotherapy in combination with radiotherapy (15,17,18,20-22) (see Tables 1 and 2).

In the study by Marchegiani et al. (15), a total of 102 patients with invasive IPMN were included. Nineteen patients $(19 \%)$ received adjuvant treatment. For the entire cohort, adjuvant therapy did not prolong survival. After subgroup analysis, it could be demonstrated that adjuvant therapy could significantly improve 5-year CSS in patients with node-positive disease and tubular differentiation.

Duconseil et al. (16) investigated the efficacy of adjuvant chemotherapy in a multicenter French study. A total of 82 patients with resected invasive IPMN were included, of whom $61(74 \%)$ received adjuvant chemotherapy. OS was significantly improved in patients who received adjuvant chemotherapy. However, the beneficial effect of adjuvant chemotherapy was limited to patients with node-positive disease.

Using the National Cancer Data Base, McMillan et al. (17) reported the largest study to date on adjuvant treatment for invasive IPMN. This registry-based study included 1,220 patients with invasive IPMN, 541 (44\%) of whom received adjuvant therapy. The results showed that adjuvant therapy significantly enhanced the OS. The survival advantage was especially prominent for patients with high AJCC stage, node-positive disease, poor histological differentiation and positive resection margins.

Caponi et al. (18) included 64 patients with invasive IPMN. Adjuvant therapy was given to 33 patients (52\%). The patients that received adjuvant therapy had significantly improved OS as well as DFS. The prognostic impact of adjuvant treatment was limited to patients with nodepositive disease.

Worni et al. (19) used the SEER registry to evaluate the prognostic role of adjuvant radiotherapy in 972 patients with invasive IPMN. Adjuvant radiotherapy was administered to 309 patients (32\%). Propensity score matching was used. It was found that adjuvant radiotherapy was associated with improved CSS in patients with high T-stage and node-positive disease.

In the study by Alexander et al. (20), 44 patients with invasive IPMN were investigated. Seventeen patients (39\%) received adjuvant treatment. For the entire cohort, adjuvant treatment was not associated with prolonged survival. 


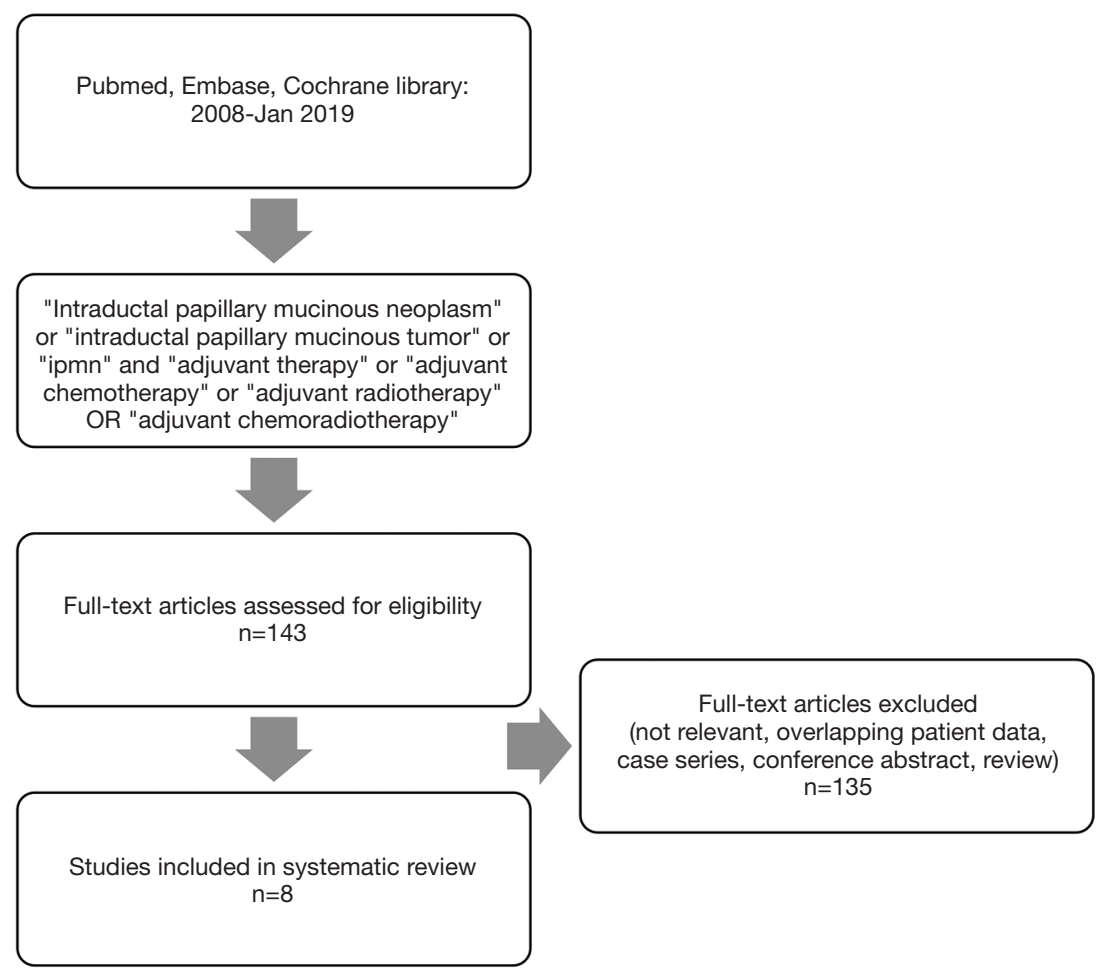

Figure 1 Search strategy.

Table 1 Study characteristics

\begin{tabular}{|c|c|c|c|c|}
\hline Reference & Country & $\mathrm{N}$ & $\begin{array}{l}\text { Proportion receiving } \\
\text { adjuvant treatment }\end{array}$ & Adjuvant treatment \\
\hline Marchegiani, 2019 (15) & Italy & 102 & $19(19 \%)$ & $\begin{array}{l}\text { Gemcitabine }(n=15) \text {, gemcitabine/oxaliplatin }(n=2) \text { or } 5-F U / \\
\text { oxaliplatin }(n=2) .5 \text { patients received additional adjuvant } \\
\text { radiotherapy (chemoradiation) }\end{array}$ \\
\hline Duconseil, 2017 (16) & France & 82 & $61(74 \%)$ & Chemotherapy \\
\hline Caponi, 2013 (18) & Italy & 64 & $33(52 \%)$ & Gemcitabine or gemcitabine/radiotherapy \\
\hline Worni, 2012 (19) & USA & 972 & $309(32 \%)$ & Radiotherapy \\
\hline Alexander, $2011(20)$ & USA & 44 & $17(39 \%)$ & Radiotherapy with $5-\mathrm{FU}$, capecitabine or 5-FU/gemcitabine \\
\hline Turrini, $2010(21)$ & USA & 98 & $37(38 \%)$ & 5-FU or gemcitabine/radiotherapy \\
\hline
\end{tabular}

5-FU, 5-fluorouracil.

However, for patients with node-positive disease, adjuvant treatment significantly improved both CSS and OS.

In the study by Turrini et al. (21), 98 patients with invasive IPMN were included. Out of these patients, $37(38 \%)$ received adjuvant treatment. There was no survival benefit of adjuvant therapy, even after stratification for nodal status. However, patients who received adjuvant treatment tended to have more advanced disease.

Swartz et al. (22) performed an analysis of 70 patients with invasive IPMN. Adjuvant therapy was administered to 
Table 2 Study outcomes

\begin{tabular}{|c|c|c|c|c|c|c|c|}
\hline Reference & Comparison & \multicolumn{6}{|c|}{ Survival benefit } \\
\hline Marchegiani, 2019 (15) & $\begin{array}{l}\text { AT vs. surgery } \\
\text { alone }\end{array}$ & No & Yes & NA & NA & NA & $\begin{array}{c}\text { Tubular } \\
\text { carcinoma }\end{array}$ \\
\hline Duconseil, 2017 (16) & $\begin{array}{c}\text { ACT vs. surgery } \\
\text { alone }\end{array}$ & Yes & Yes & NA & NA & NA & NA \\
\hline McMillan, 2016 (17) & $\begin{array}{l}\text { AT vs. surgery } \\
\text { alone }\end{array}$ & Yes & Yes & Stage II-IV & Yes & $\begin{array}{c}\text { Poor } \\
\text { differentiation }\end{array}$ & NA \\
\hline Caponi, 2013 (18) & $\begin{array}{l}\text { AT vs. surgery } \\
\text { alone }\end{array}$ & Yes & Yes & NA & NA & NA & NA \\
\hline Turrini, 2010 (21) & $\begin{array}{l}\text { AT vs. surgery } \\
\text { alone }\end{array}$ & No & No & No & No & NA & NA \\
\hline Swartz, 2010 (22) & $\begin{array}{c}\text { AT vs. surgery } \\
\text { alone }\end{array}$ & Yes & Yes & NA & Yes & NA & NA \\
\hline
\end{tabular}

ACT, adjuvant chemotherapy; AR, adjuvant radiotherapy; AT, adjuvant therapy; NA, not available.

40 patients $(57 \%)$. The receipt of adjuvant treatment was associated with improved OS. Specific benefit was noted for those with node-positive disease and positive resection margins.

\section{Discussion}

The efficacy of adjuvant therapy for invasive IPMN is not well defined. Treatment decisions are often extrapolated from studies in PDAC where randomized trials have generated beneficial results (11-13). To our knowledge, this is the first systematic review on the role of adjuvant therapy for invasive IPMN. The current study demonstrates a significant survival benefit from adjuvant therapy compared with surgery alone for patients with invasive IPMN.

The survival benefit was most prominent among patients with node-positive disease (15-20,22). This observation suggests that lymph node status should be considered when administrating adjuvant therapy for patients with invasive IPMN.

Other conventional histopathological factors were also found to impact the response to adjuvant therapy in individual studies. For example, patients with positive resection margins $(17,22)$ or poorly differentiated tumors $(17)$ derived a clear benefit from adjuvant treatment.

As the knowledge concerning IPMN increases, more data will emerge regarding the natural history and behavior of IPMN. Invasive IPMN can be subclassified into tubular, colloid and oncocytic subtypes (2). Interestingly, these subtypes may have varying response to adjuvant treatment. For example, in the study by Marchegiani et al. (15), a survival benefit was only seen in those with a tubular subtype.

The colloid subtype of IPMN seems to have a distinct biology and typically arises from intestinal differentiation $(1,23)$. The oncocytic subtype was not included in any of the studies. Although rare (9), the oncocytic subtype may, in similarity to colloid carcinoma, show differing response to chemotherapy when compared to the tubular subtype.

In several studies, the efficacy of adjuvant therapy was not noticed in univariate analyses or when the entire cohort was evaluated. These discrepancies may be influenced by selection bias as patients with advanced histopathology were more prone to receive adjuvant therapy. This is a known issue in retrospective analysis of adjuvant treatment. The selective administration of adjuvant therapy for patients with adverse oncological characteristics has been noted in PDAC and may bias any retrospective analysis attempting 
to measure the benefit of adjuvant treatment (24).

This study was limited by several factors. Although two studies $(17,19)$ used large patient cohorts, most selected studies were characterized by a limited sample size. Another limitation was the retrospective nature of the studies, leading to potential selection bias. The adjuvant treatment protocols also varied, where chemotherapy or radiotherapy were used either alone or in combination.

\section{Conclusions}

The findings from the present study suggest that patients with invasive IPMN may derive benefit from adjuvant treatment. The survival effects are especially clear for those with advanced tumor characteristics and tubular phenotype. Prospective, randomized trials are needed to confirm these findings and to optimize adjuvant treatment protocols in patients with invasive IPMN.

\section{Acknowledgments}

None.

\section{Footnote}

Conflicts of Interest: The authors have no conflicts of interest to declare.

Ethical Statement: The authors are accountable for all aspects of the work in ensuring that questions related to the accuracy or integrity of any part of the work are appropriately investigated and resolved.

\section{References}

1. Adsay N, Kloppel G, Fukushima N. WHO Classification of Tumors, Intraductal neoplasms of the pancreas. Lyon, France, WHO Press 2010.

2. Adsay V, Mino-Kenudson M, Furukawa T, et al. Pathologic Evaluation and Reporting of Intraductal Papillary Mucinous Neoplasms of the Pancreas and Other Tumoral Intraepithelial Neoplasms of Pancreatobiliary Tract: Recommendations of Verona Consensus Meeting. Ann Surg 2016;263:162-77.

3. European Study Group on Cystic Tumours of the Pancreas. European evidence-based guidelines on pancreatic cystic neoplasms. Gut 2018;67:789-804.

4. Tanaka M, Fernandez-Del Castillo C, Kamisawa T, et al. Revisions of international consensus Fukuoka guidelines for the management of IPMN of the pancreas. Pancreatology 2017;17:738-53.

5. Aronsson L, Andersson R, Ansari D. Intraductal papillary mucinous neoplasm of the pancreas - epidemiology, risk factors, diagnosis, and management. Scand J Gastroenterol 2017;52:803-15.

6. Tan MC, Basturk O, Brannon AR, et al. GNAS and KRAS Mutations Define Separate Progression Pathways in Intraductal Papillary Mucinous Neoplasm-Associated Carcinoma. J Am Coll Surg 2015;220:845-54.e1.

7. Adsay NV, Merati K, Nassar H, et al. Pathogenesis of colloid (pure mucinous) carcinoma of exocrine organs: Coupling of gel-forming mucin (MUC2) production with altered cell polarity and abnormal cell-stroma interaction may be the key factor in the morphogenesis and indolent behavior of colloid carcinoma in the breast and pancreas. Am J Surg Pathol 2003;27:571-8.

8. Adsay NV, Merati K, Basturk O, et al. Pathologically and biologically distinct types of epithelium in intraductal papillary mucinous neoplasms: delineation of an "intestinal" pathway of carcinogenesis in the pancreas. Am J Surg Pathol 2004;28:839-48.

9. Mino-Kenudson M, Fernandez-del Castillo C, Baba Y, et al. Prognosis of invasive intraductal papillary mucinous neoplasm depends on histological and precursor epithelial subtypes. Gut 2011;60:1712-20.

10. Waters JA, Schnelldorfer T, Aguilar-Saavedra JR, et al. Survival after resection for invasive intraductal papillary mucinous neoplasm and for pancreatic adenocarcinoma: a multi-institutional comparison according to American Joint Committee on Cancer Stage. J Am Coll Surg 2011;213:275-83.

11. Neoptolemos JP, Stocken DD, Friess H, et al. A randomized trial of chemoradiotherapy and chemotherapy after resection of pancreatic cancer. $\mathrm{N}$ Engl J Med 2004;350:1200-10.

12. Neoptolemos JP, Stocken DD, Bassi C, et al. Adjuvant chemotherapy with fluorouracil plus folinic acid vs gemcitabine following pancreatic cancer resection: a randomized controlled trial. JAMA 2010;304:1073-81.

13. Neoptolemos JP, Palmer DH, Ghaneh P, et al. Comparison of adjuvant gemcitabine and capecitabine with gemcitabine monotherapy in patients with resected pancreatic cancer (ESPAC-4): a multicentre, open-label, randomised, phase 3 trial. Lancet 2017;389:1011-24.

14. Moher D, Shamseer L, Clarke M, et al. Preferred reporting items for systematic review and meta-analysis 
protocols (PRISMA-P) 2015 statement. Syst Rev 2015;4:1.

15. Marchegiani G, Andrianello S, Dal Borgo C, et al. Adjuvant chemotherapy is associated with improved postoperative survival in specific subtypes of invasive intraductal papillary mucinous neoplasms (IPMN) of the pancreas: it is time for randomized controlled data. HPB (Oxford) 2019;21:596-603.

16. Duconseil P, Perinel J, Autret A, et al. Resectable invasive IPMN versus sporadic pancreatic adenocarcinoma of the head of the pancreas: Should these two different diseases receive the same treatment? A matched comparison study of the French Surgical Association (AFC). Eur J Surg Oncol 2017;43:1704-10.

17. McMillan MT, Lewis RS, Drebin JA, et al. The efficacy of adjuvant therapy for pancreatic invasive intraductal papillary mucinous neoplasm (IPMN). Cancer 2016;122:521-33.

18. Caponi S, Vasile E, Funel N, et al. Adjuvant chemotherapy seems beneficial for invasive intraductal papillary mucinous neoplasms. Eur J Surg Oncol 2013;39:396-403.

19. Worni M, Akushevich I, Gloor B, et al. Adjuvant radiotherapy in the treatment of invasive intraductal papillary mucinous neoplasm of the pancreas: an analysis

Cite this article as: Aronsson L, Marinko S, Ansari D, Andersson R. Adjuvant therapy in invasive intraductal papillary mucinous neoplasm (IPMN) of the pancreas: a systematic review. Ann Transl Med 2019;7(22):689. doi: 10.21037/atm.2019.10.37 of the surveillance, epidemiology, and end results registry. Ann Surg Oncol 2012;19:1316-23.

20. Alexander BM, Fernandez-Del Castillo C, Ryan DP, et al. Intraductal papillary mucinous adenocarcinoma of the pancreas: clinical outcomes, prognostic factors, and the role of adjuvant therapy. Gastrointest Cancer Res 2011;4:116-21.

21. Turrini O, Waters JA, Schnelldorfer T, et al. Invasive intraductal papillary mucinous neoplasm: predictors of survival and role of adjuvant therapy. HPB (Oxford) 2010;12:447-55.

22. Swartz MJ, Hsu CC, Pawlik TM, et al. Adjuvant chemoradiotherapy after pancreatic resection for invasive carcinoma associated with intraductal papillary mucinous neoplasm of the pancreas. Int J Radiat Oncol Biol Phys 2010;76:839-44.

23. Ban S, Naitoh Y, Mino-Kenudson M, et al. Intraductal papillary mucinous neoplasm (IPMN) of the pancreas: its histopathologic difference between 2 major types. Am J Surg Pathol 2006;30:1561-9.

24. Russ AJ, Weber SM, Rettammel RJ, et al. Impact of selection bias on the utilization of adjuvant therapy for pancreas adenocarcinoma. Ann Surg Oncol 2010;17:371-6. 